\title{
Recours a la Médecine Traditionnelle chez les Rhumatisants en Guinée
}

Kamissoko Aly Badra, (MD, CCA)

Université Gamal Abdel Nasser, Hôpital National Ignace Deen, Conakry, Guinée

Traoré Marie, (MD)

Service de Rhumatologie, Hôpital Sud Francilien, Corbeil Essonne, France

Wann Thierno Amadou, (MD, CCA)

Baldé Mamadou Dian, (MD)

Université Gamal Abdel Nasser,

Hôpital National Ignace Deen, Conakry, Guinée

Diallo Mamadou Lamine, (MD)

Université Kofi Annan, Hôpital National Ignace Deen, Conakry, Guinée

Oniankitan Sadat, (MD)

Université de Lomé, CHU Sylvanus Olympio, Lomé, Togo

Camara Alpha Ketty, (MD)

Awada Mohamed, (MD, MA)

Université Gamal Abdel Nasser,

Hôpital National Ignace Deen, Conakry, Guinée

Diallo Alhassane, (MD, PhD)

INSERM, U1137-IAME, Département de biostatistique, investigations

cliniques et pharmaceutiques des maladies infectieuses, Université Paris

Diderot, Paris, France

Oniankitan Owonayo, (MD, Prof.)

Université de Lomé, CHU Sylvanus Olympio, Lomé, Togo

Doi:10.19044/esj.2019.v15n24p32～ＵRL:http://dx.doi.org/10.19044/esj.2019.v15n24p32

\section{Résumé}

Objectif : Déterminer la fréquence et le profil de l'utilisation de la médecine traditionnelle chez les patients souffrant de rhumatisme à l'hôpital national Ignace Deen de Conakry (Guinée). Patients et méthodes : Etude transversale de type descriptif dans le service de rhumatologie de l'HNID entre janvier et mars 2018. Résultats : Sur 108 patients interrogés, 40 avaient eu recours à la médecine traditionnelle soit $37,03 \%$. On notait une prédominance féminine ( 21 femmes ; 52,5\%) avec un sexe ratio de $0,90 \mathrm{H} / \mathrm{F}$. L'âge moyen des patients était de 44,75 $\pm 17,24$ ans. La polyarthrite rhumatoïde et la 
gonarthrose étaient les principales affections rhumatologiques observées avec respectivement $41,86 \%$ et $16,27 \%$. Le délai diagnostique était de $6,54 \pm 1,25$ ans. La nature du traitement utilisé était dominée par la prise de décoction $(57,50 \%)$ et le motif d'utilisation du traitement était de soulager la douleur (85\%). La voie orale était le principal mode d'utilisation (67,5\%). Plus de la moitié des patients $(52,50 \%)$ n'étaient pas satisfaits du résultat du traitement traditionnel reçu et ne connaissaient pas le nom du traitement utilisé dans leur langue vernaculaire. Ils pensaient dans $47,50 \%$ des cas que le traitement traditionnel entrainait des effets indésirables et avait de possibles interactions avec le traitement médicamenteux moderne. Conclusion : Plus du tiers des patients consultant en rhumatologie ont recours à la médecine traditionnelle même s'ils croient qu'elle a des effets indésirables. Ce phénomène entraîne un retard à la consultation chez le rhumatologue, d'où la nécessité d'une meilleure communication entre les différents acteurs.

Mots clés: Maladies rhumatismales, médecine traditionnelle, relation médecin-patient, Guinée 


\title{
Use of Traditional Medicine in Rheumatism Patients in Guinea
}

\section{Kamissoko Aly Badra, (MD, CCA)}

Université Gamal Abdel Nasser, Hôpital National Ignace Deen, Conakry, Guinée

Traoré Marie, (MD)

Service de Rhumatologie, Hôpital Sud Francilien, Corbeil Essonne, France

Wann Thierno Amadou, (MD, CCA)

Baldé Mamadou Dian, (MD)

Université Gamal Abdel Nasser, Hôpital National Ignace Deen, Conakry, Guinée

Diallo Mamadou Lamine, (MD)

Université Kofi Annan, Hôpital National Ignace Deen, Conakry, Guinée

Oniankitan Sadat, (MD)

Université de Lomé, CHU Sylvanus Olympio, Lomé, Togo

Camara Alpha Ketty, (MD)

Awada Mohamed, (MD, MA)

Université Gamal Abdel Nasser, Hôpital National Ignace Deen, Conakry, Guinée

Diallo Alhassane, (MD, PhD)

INSERM, U1137-IAME, Département de biostatistique, investigations cliniques et pharmaceutiques des maladies infectieuses, Université Paris Diderot, Paris, France

Oniankitan Owonayo, (MD, Prof.)

Université de Lomé, CHU Sylvanus Olympio, Lomé, Togo

\begin{abstract}
Aim: To determine the frequency and pattern of use of traditional medicine in rheumatic patients at Ignace Deen National Hospital in Conakry, Guinea. Patients and methods: Descriptive cross-sectional study in the rheumatology department of HNID between January and March 2018 Results: Of the 108 patients interviewed, 40 had used traditional medicine (37.03\%). There was a female predominance (21 women, $52.5 \%$ ) with a sex ratio of 0.90 . The mean age of the patients was $44.75 \pm 17.24$ years. Rheumatoid arthritis and knee osteoarthritis were the main rheumatic diseases observed with $41.86 \%$ and $16.27 \%$ respectively. The diagnostic delay was
\end{abstract}


$6.54 \pm 1.25$ years. The nature of the treatment used was dominated by taking a decoction $(57.50 \%)$ and the reason for using traditional treatment was to relieve pain $(85 \%)$. The oral route was the main mode of use of traditional treatment $(67.5 \%)$. More than half of the patients $(52.50 \%)$ were not satisfied with the outcome of traditional treatment received and did not know the name of the treatment used in their vernacular language. In $47.50 \%$ of cases they thought that traditional treatment had adverse effects and had possible interactions with modern drug therapy. Conclusion: More than a third of rheumatology consulting patients use traditional medicine even though they believe it has side effects. This phenomenon leads to a delay in consultation with the rheumatologist, hence the need for better communication between the different actors.

Keywords: Rheumatic diseases, traditional medicine, doctor-patient relationship, Guinea

\section{Introduction}

L'organisation mondiale de la santé (OMS) définit la médecine traditionnelle comme «l'ensemble des connaissances, compétences et pratiques fondées sur les théories, les croyances et les expériences propres aux différentes cultures, explicables ou non, utilisées dans le maintien de la santé, comme dans la prévention, le diagnostic, l'amélioration ou le traitement de la maladie physique et mentale » (Organisation Mondiale de la Santé, 2002). La fréquence d'utilisation de la médecine traditionnelle varie d'un pays à un autre (75\% en France, $71 \%$ au Chili, 28,36\% en Afrique du nord, 80\% en Afrique de l'ouest) (Bussman RW et al., 2013; Hmamouchi I et al., 2012; Organisation Mondiale de la Santé, 2002.). Notre étude avait pour objectif de déterminer la fréquence et le profil de l'utilisation de la médecine traditionnelle chez les patients souffrant de rhumatisme à l'hôpital national Ignace Deen de Conakry (Guinée).

\section{Patients et méthodes:}

Nous avons mené une étude transversale de type descriptif à l'aide d'un questionnaire préétabli dans le service de rhumatologie de l'hôpital national Ignace Deen entre janvier et mars 2018. Les patients diagnostiqués d'une pathologie rhumatismale ont été interrogés de façon consécutive. Le questionnaire était administré aux patients directement à la fin de la consultation avec le rhumatologue. Les patients n'ayant pas accepté de participer à l'étude et ceux ne répondant pas aux critères diagnostiques des affections rhumatismales n'ont pas été inclus. Le questionnaire comportait :

- les caractéristiques sociodémographiques (âge, sexe, profession, provenance, niveau d'instruction et le statut matrimonial) 
- $\quad$ les caractéristiques cliniques (motif de consultation, antécédents, durée d'évolution de la maladie, date de consultation, diagnostic).

- $\quad$ les caractéristiques d'utilisation de la médecine traditionnelle (raison d'utilisation, type de traitement traditionnel utilisé, fréquence d'utilisation, efficacité, connaissance des effets secondaires et des possibles interactions médicamenteuses avec les produits pharmacologiques). Pour les malades n'ayant pas eu recours à la médecine traditionnelle, la raison ne leur avait pas été demandée ; ils n'avaient pas été soumis à la troisième partie du questionnaire. Les variables quantitatives ont été exprimées en moyennes \pm écart-type, les variables qualitatives ont été exprimées en pourcentage.

\section{Considérations éthiques}

Le consentement éclairé des patients a été obtenu et le protocole accepté par le comité d'éthique de l'Hôpital Ignace Deen.

\section{Résultats}

Sur 108 patients interrogés, $40(37,03 \%)$ avaient eu recours à la médecine traditionnelle. On notait une prédominance féminine $(21$ femmes ; $52,5 \%$ ) avec un sexe ratio de $0,90 \mathrm{H} / \mathrm{F}$ (homme/femme). La tranche d'âge la plus représentée était celle de 50-59 ans avec un âge moyen de 44,75 \pm 17,24 ans. $42,50 \%$ des patients ne savaient ni lire ni écrire (Tableau I). Les motifs de consultation étaient dominés par la polyarthrite (30, 43\%). Les pathologies inflammatoires étaient les plus représentées avec 67,50\%. La polyarthrite rhumatoïde et la gonarthrose étaient les principales affections rhumatologiques observées avec respectivement $41,86 \%$ et $16,27 \%$. Le délai diagnostique était de 6,54 $\pm 1,25$ ans (Tableau II). Les patients utilisaient principalement des traitements traditionnels à base de décoction $(57,50 \%)$ et le motif d'utilisation était de soulager la douleur chez $85 \%$. La voie orale était le principal mode d'utilisation du traitement traditionnel (67,5\%). Plus de la moitié des patients $(52,50 \%)$ n'étaient pas satisfaits du résultat du traitement traditionnel reçu et ne connaissaient pas le nom du traitement utilisé dans leur langue vernaculaire. Ils pensaient dans $47,50 \%$ des cas que le traitement traditionnel entraine des effets indésirables et disaient savoir l'existence de possibles interactions entre le traitement traditionnel et le traitement médicamenteux moderne (Tableau III). 
Tableau I : Répartition des patients selon les caractéristiques sociodémographiques

\begin{tabular}{|c|c|c|}
\hline & $\begin{array}{l}\text { Effectif } \\
\mathrm{N}=40\end{array}$ & $\begin{array}{l}\text { Moyenne } \pm \text { ET* ou } \\
\text { Pourcentage }(\%)\end{array}$ \\
\hline Sexe (féminin) & 21 & 52,25 \\
\hline Age (ans) & $\mathrm{n}=40$ & $44,75 \pm 17,24$ \\
\hline \multicolumn{3}{|c|}{ Situation matrimoniale } \\
\hline Marié (e) & 26 & 65 \\
\hline Célibataire & 8 & 20 \\
\hline Veuf (ve) & 5 & 12,5 \\
\hline Divorcé (e) & 1 & 2,5 \\
\hline \multicolumn{3}{|c|}{ Catégorie socio-professionnelle } \\
\hline Profession libérale & 15 & 37,5 \\
\hline Ménagère & 11 & 27,5 \\
\hline Fonctionnaire & 8 & 1,0 \\
\hline Etudiants/Elèves & 6 & 15,0 \\
\hline \multicolumn{3}{|l|}{ Provenance } \\
\hline Urbaine & 21 & 52,5 \\
\hline Rurale & 19 & 47,5 \\
\hline \multicolumn{3}{|c|}{ Niveau d'instruction } \\
\hline Non scolarisé & 17 & 42,50 \\
\hline Supérieur & 9 & 32,41 \\
\hline Secondaire & 6 & 15,00 \\
\hline Ecole coranique & 6 & 15,0 \\
\hline Primaire & 2 & 5,0 \\
\hline Total & 40 & 100 \\
\hline
\end{tabular}

Tableau II: Caractéristiques cliniques des patients

\begin{tabular}{lll}
\hline & \multicolumn{1}{c}{$\begin{array}{c}\text { Effectif } \\
\mathbf{N}=\mathbf{4 0}\end{array}$} & \multicolumn{1}{c}{$\begin{array}{c}\text { Moyenne } \mathbf{E} \text { ET* } \\
\text { Pourcentage (\%) }\end{array}$} \\
\hline $\begin{array}{l}\text { Délai diagnostique (ans) } \\
\text { Pathologies inflammatoires }\end{array}$ & $\mathrm{n}=40$ & $6,54 \pm 1,25$ \\
Pathologies mécaniques & 13 & 67,5 \\
Motifs de consultation & & 32,5 \\
Polyarthrite & 14 & \\
Lombalgie & 9 & 30,43 \\
Gonalgie & 7 & 22,5 \\
Polyarthralgie & 5 & 15,22 \\
Cervicalgie & 3 & 10,09 \\
Autres** & 10 & 6,25 \\
Diagnostic & & 21,70 \\
Polyarthrite rhumatoïde & 18 & \\
Gonarthrose & 7 & 41,86 \\
Spondylarthrite ankylosante & 5 & 16,27 \\
Lombarthrose & 5 & 11,62 \\
Autres*** & 8 & 11,62 \\
:ET : & & 18,60 \\
\hline
\end{tabular}

*ET : écart-type

**Autres : deux cas de fessalgie, deux cas de gonarthrite et deux cas de douleur d'épaule ; un cas de talalgie et un cas de douleur de hanche. 
***Autres : un cas de chacune des pathologies suivantes : arthrite juvénile idiopathique, lupus érythémateux systémique, goutte, rhumatisme inflammatoire indifférencié, ostéoporose, ostéonécrose de la tête fémorale, coxarthrose et capsulite rétractile.

Tableau III: Caractéristiques d'utilisation des traitements traditionnels

\begin{tabular}{lll}
\hline & Effectif & Pourcentage (\%) \\
\hline But d'usage du traitement traditionnel & & \\
Soulager la douleur & 36 & 85,0 \\
Guérir la maladie & 4 & 15,0 \\
Nature du traitement utilisé & 23 & 57,5 \\
Décoction & 3 & 7,5 \\
Ecorce de plante & 3 & 7,5 \\
Viande de chacal & 3 & 7,5 \\
Acupuncture & 2 & 5,0 \\
Kaolin & 2 & 5,0 \\
Poudre & 2 & 5,0 \\
Versets coraniques & 2 & 5,0 \\
Infusion & 1 & 2,5 \\
Macération & 1 & 2,5 \\
Viande de chèvre & 1 & 2,5 \\
Mode d'utilisation du traitement & & \\
traditionnel & & \\
Voie orale & 17 & 42,5 \\
Aiguille sur le corps & 11 & 27,5 \\
Lavement & 8 & 20,0 \\
Incantation & 4 & 10,0 \\
Perception sur l'efficacité du & & \\
traitement & & 52,5 \\
Aggravation & 21 & 42,5 \\
Stabilisation & 17 & 5,0 \\
Amélioration & 2 & 55,0 \\
Information du rhumatologue & 22 & \\
Possibilité d'interactions & & 47,5 \\
médicamenteuses selon le patient & & 22,5 \\
Oui & 19 & \\
Non & 12 & \\
Ne sait pas & 9 & \\
\hline & & \\
& & \\
\hline
\end{tabular}

\section{Discussion}

Notre étude a eu trait à l'utilisation de la médecine traditionnelle par les patients consultant en rhumatologie. Les patients souffrant aussi bien de maladies inflammatoires que mécaniques avaient recours à la médecine traditionnelle. Le recours à la médecine traditionnelle est une pratique fréquente. La prévalence de $37.03 \%$ de recours à la médecine traditionnelle est supérieure aux données du Maroc où on retrouve 28,6 \% de cas d'utilisation de plantes médicinales en rhumatologie (Hmamouchi I et al., 2012). D'autres études montrent des chiffres plus importants car elles prennent en compte une définition plus large de la médecine traditionnelle (phytothérapie, les points de feu, les saignées, l'acupuncture, les techniques 
de relaxation, la chiropractie et les cures de désintoxication) (Rao JK et al., 1999; Kestin M et al., 1985). Une prédominance féminine est retrouvée dans la littérature (Hmamouchi I et al., 2012). Nous avons également retrouvé plus de femmes que d'hommes. La prédominance des femmes dans l'épidémiologie des maladies rhumatismales pourrait l'expliquer.

L'âge moyen de nos patients était de 44,75 $\pm 17,24$ ans. Ce résultat est proche de celui retrouvé au Maroc (49 ans) (Belkhou A et al., 2012). Plusieurs autres études africaines ont rapporté des résultats similaires (Hmamouchi I et al., 2012; Annick K et al., 2016) liés probablement à la jeunesse de la population africaine.

La médecine traditionnelle reste beaucoup implantée dans les milieux ruraux en Afrique (60\% au Maroc) (Hmamouchi I et al., 2012). Ce phénomène s'explique par la proximité géographique et culturelle des patients et des tradipraticiens (Adjet AA et al., 2016). Cependant 72,5\% des patients habitaient en zone urbaine. Ce résultat a été influencé par la situation géographique de notre site de recrutement (service de rhumatologie à Conakry), mais aussi par les moyens modernes de communication désormais utilisés par les tradipraticiens (Koua AK et al., 2016).

Nos patients ne savaient ni lire ni écrire dans $42,50 \%$, ils étaient sans emploi dans 37,5\%. Le taux d'analphabétisme est élevé en Guinée (INS, 2018). Cette couche est plus encline aux traitements traditionnels car moins informée sur la médecine moderne.

Les pathologies inflammatoires étaient les plus représentées avec 27 (62, 79\%). Par contre l'étude réalisée en Allemagne (Flammersfeld L et al., 2015) rapporte une fréquence élevée des douleurs rachidiennes (79\%). Dans notre contexte les rhumatismes inflammatoires sont fréquents (Kamissoko AB et al., 2018). Ces rhumatismes parfois déformants sont souvent associés à des causes surnaturelles par les patients et motivent ainsi le recours à la médecine traditionnelle.

La voie orale et le lavement étaient les principaux modes d'utilisation du traitement traditionnel avec respectivement 27 cas $(67,5 \%)$ et 9 cas $(22,5 \%)$. Ce résultat est identique à celui retrouvé au Ghana et en Ethiopie ( respectivement 77\% et 44\%) (Boadu AA et al., 2017; Wubetu M et al., 2010). Ce résultat pourrait s'expliquer par le fait que les tradipraticiens préfèrent les voies simples en raison de leur incapacité à administrer des remèdes dans d'autres voies comme celle intraveineuse. Le recours à la médecine traditionnelle était motivé dans $85 \%$ par le soulagement de la douleur. Ce résultat était en accord avec les données de Rao et al. (Rao JK et al., 1999). Ceci est lié à la symptomatologie douloureuse prédominante des pathologies rhumatismales. La moitié de nos patients (50\%) ne connaissaient pas le nom du traitement utilisé dans leur langue vernaculaire. Ce qui peut être expliqué par le fait que les traitements traditionnels ne soient pas bien codifiés et 
harmonisés. Plus de la moitié des patients (55\%) n'avaient pas informé leur rhumatologue d'avoir eu recours au traitement traditionnel. Des proportions similaires ont été rapportées dans la littérature (Hmamouchi I et al. 2012; Eseinberg et al., 1993; Elder NC et al., 1997; Visser GJ et al., 1992). Ceci s'explique par le fait que les patients évitent les réactions négatives des médecins qui n'apprécient pas ces traitements non médicaux. En effet la moitié de nos patients estimaient que le traitement traditionnel peut entrainer des effets indésirables et de possibles interactions avec le traitement médicamenteux moderne. Parmi nos patients, 52,50\% n'étaient pas satisfaits du traitement traditionnel. Par contre, une étude réalisée en Ethiopie (Yared A et al., 2017) avait montré que 79,4\% des personnes interrogées étaient satisfaites. On pourrait expliquer cette insatisfaction de nos patients par le caractère chronique des rhumatismes qui évoluent le plus souvent par poussées et rémissions. Au terme de cette analyse, il ressort qu'avant la consultation en rhumatologie, les patients ont fréquemment recours à la médecine traditionnelle pour soulager leur douleur.

\section{Conclusion}

Plus du tiers des patients consultant en rhumatologie ont recours à la médecine traditionnelle même s'ils croient qu'elle a des effets indésirables. Ce phénomène entraîne un retard à la consultation chez le rhumatologue, d'où la nécessité d'une meilleure communication entre les différents acteurs.

\section{References:}

1. Adjet AA, Adou DL, Danièle OK. (2016). Itinéraires thérapeutiques pluriels et recours tardif des malades de l'ulcère de Buruli dans les centres de prise en charge dans le district sanitaire de Yamoussoukro (Côte d'Ivoire). ESJ. 12(30) : 263-279.

2. Belkhou A, Cherquaoui H, EL Hassani S. (2012). Le retard au diagnostic et au traitement du patient rhumatisant : Quels déterminants ? Enquête transversale aux secteurs publique et privé à Marrakech. Rev Mar Rhum ; 20 :38-41.

3. Boadu AA et Asase A. (2017). Documentation of Herbal Medicines Used for the Treatment and Management of Human Diseases by Some Communities in Southern Ghana. Evid Based Complement Alternat Med. 3043061. doi:10.1155/2017/3043061

4. Bussmann RW. (2013). The globalization of traditional medicine in northern peru: from shamanism to molecules. Evid Based Complement Alternat Med. 291903. doi: 10.1155/2013/291903

5. Institut national de la statistique de Guinée (INS) http://www.statguinee.org/ consuté le 29 décembre 2018. 
6. Eisenberg DM, Kessler RC, Foster C, Norlock FE, Calkins DR, Delbanco TL. (1993). Unconventional medicine in the United States. Prevalence, costs, and patterns of use. N Engl J Med; 328:246-52.

7. Elder NC, Gillcrist A, Minz R. (1997). Use of alternative health care by family Practice patients. Arch Fam Med ; 6: 81-4.

8. Flammersfeld L, Weinmann B. (2005). Traitement du rhumatisme par l'extrait sec de racine d'harpagophytum. Une étude multicentrique en cabinet médical. Phytothérapie ; 3 : 104-106.

9. Hmamouchi I et al. (2012). Pratique traditionnelle d'utilisation des plantes médicinales marocaines en rhumatologie. Rev Mar Rhum; 22: 52-6.

10. Kamissoko AB et al. (2018). Panorama des pathologies rhumatismales à Conakry. ESJ; 14 (24): 422-431.

11. Kestin M, Miller L, Littlejohn G, Wahlqvist M. (1985). The use of unproven remedies for rheumatoid arthritis in Australia. Med J Aust ; 143(11):516-8.

12. Koua AK, Yao LY, N'Goran GK. (2016). Insertion des jeunes déscolarisés dans la médecine traditionnelle en Côte d'Ivoire : activation des ressources relationnelles et idéologiques comme stratégie. ESJ. 12 (30) : 188-199

13. Koulibaly A, Monian M, Ackah J AAB, Koné MW, Traoré K. (2016). Étude ethnobotanique des plantes médicinales : cas des affections les plus fréquentes d'une région agricole Daloa (Centre Ouest, Côte d'Ivoire). JAPS ; 31 : 5021-5032

14. Organisation Mondiale de la Santé (OMS) (2002). Stratégie de l'OMS pour la médecine traditionnelle pour 2002-2005, WHO/EDM/TRM/2002, Genève: 65

15. Rao JK, Mihaliak K, Kroenke K, Bradley J, Tierney WM, Weinberger M. (1999). Use of complementary therapies for arthritis among patients of rheumatologists. Ann Intern Med; 131:409-16.

16. Visser GJ, Peters L, Rasker JJ. (1992). Rheumatologists and their patients who Seek alternative care: an agreement to disagree. $\mathrm{Br} \mathrm{J}$ Rheumatol ; 31 :485-90.

17. Wubetu M, Abula T, et Dejenu G. (2017). Enquête. ethnopharmacologique sur les plantes médicinales utilisées pour traiter les maladies humaines par les médecins traditionnels dans le district de Dega Damot, Amhara, nord-ouest de l'Ethiopie. BMC Res Notes; 10: 157 .

18. Yared A. (2017). Predictors of traditional medical practices in illness behavior in northwestern Ethiopia: an integrated model of behavioral prediction based logistic regression analysis. Evid Based Complement Alternat Med. ; 4186064. doi:10.1155/2017/4186064 